\title{
From the FDA
}

Iodinated contrast agents carry revised warning labels

Labels warning of the possibility of blood clotting now appear on package inserts accompanying nonionic and ionic contrast media used in angiography.

The label change is in response to some reports of clotting that can occur when blood remains in contact with syringes containing nonionic contrast media. While coagulation in vitro occurs less often with ionic contrast media, healthcare personnel should avoid exposing blood to syringes containing ionic contrast media for prolonged periods.

Meticulous administration techniques - careful guidewire, catheter manipulation; use of manifold systems or three-way stopcocks, or both; and minimal time limitations - are a must in angiography. In addition, plastic, rather than glass, syringes may reduce the likelihood of in vitro clotting if blood enters the syringe.

Although thromboembolic events have occurred in patients undergoing angiography regardless of which contrast medium was used, researchers cannot determine if atherosclerosis or blood clots cause such events.

Pentamidine in aerosolized form receives approval

An estimated 50,000 AIDS patients could benefit from treatment with the aerosolized ver- sion of pentamidine (NubuPent). Recently approved by the FDA for treatment of recurring Pneumocystis carinii (PCP) infections, aerosolized pentamidine can be used as a preventive measure as well.

In a trial conducted by the San Francisco Community Consortium, the drug was found most effective when administered through a Respirgard II nebulizer at a dose of $300 \mathrm{mg}$ every 4 weeks. The incidence of PCP was reduced, not eliminated, among patients receiving this drug.

Carboplatin sanctioned as ovarian cancer therapy

The FDA has approved carboplatin (Paraplatin) for treating patients who, despite having undergone previous chemotherapy, including cisplatin therapy, have recurring ovarian carcinoma. In two separate trials involving 46 patients, six complete clinical responses occurred, which lasted 45 weeks to more than 71 weeks.

\section{Preventive services guide available}

A review of preventive interventions for more than 60 diseases and other conditions is available in a single source. The Guide to Clinical Preventive Services makes recommendations for immunizations and chemoprophylactic use and suggests screening and counseling interventions.
Healthcare personnel may purchase this report of the US Preventive Services Task Force, which was composed of 20 nongovernment experts from medicine and related fields, by calling Williams \& Wilkins at 1-800638-0672.

\section{FDA concurs with NIH blood transfusion guidelines}

Recent advances in the use of blood components make whole blood transfusions unnecessary most of the time, according to a National Institutes of Health $(\mathrm{NIH})$ report. The FDA concurs with this report, which details clinical scenarios in which transfusions of red cells, platelets, and fresh frozen plasma are warranted. Single copies of Transfusion Alert: Indications for the Use of Red Blood Cells, Platelets, and Fresh Frozen Plasma can be obtained from the National Blood Resource Education Program Information Center, 4733 Bethesda Ave, Suite 530, Bethesda, MD 20814. 


\section{AMAPROX DS. refier for

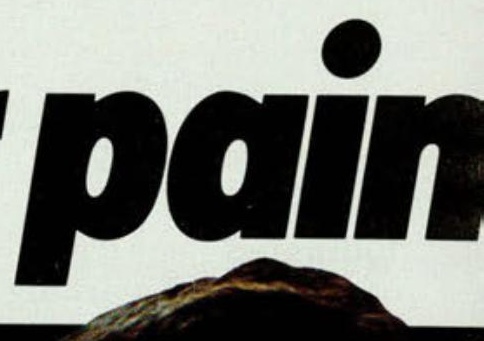

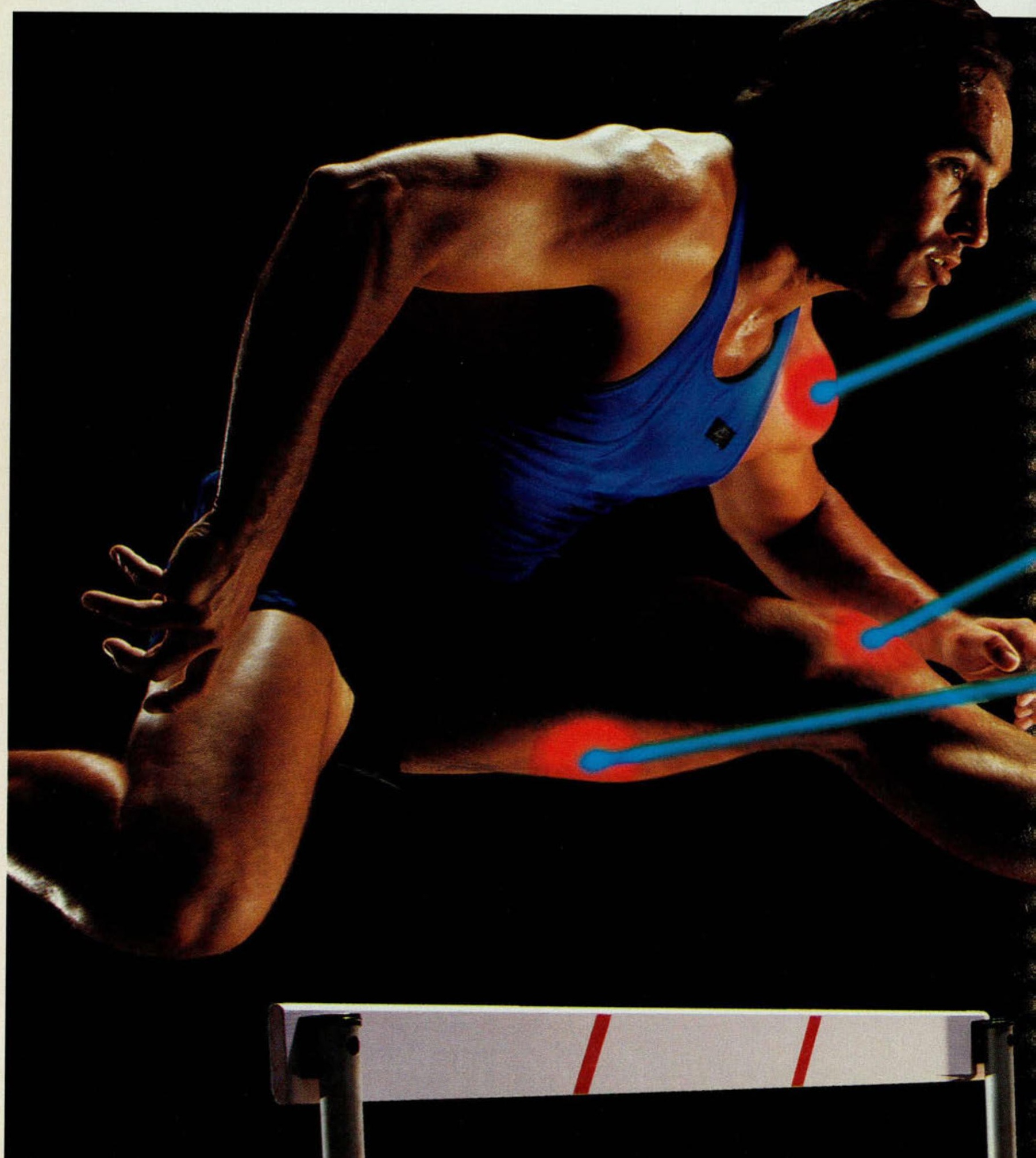




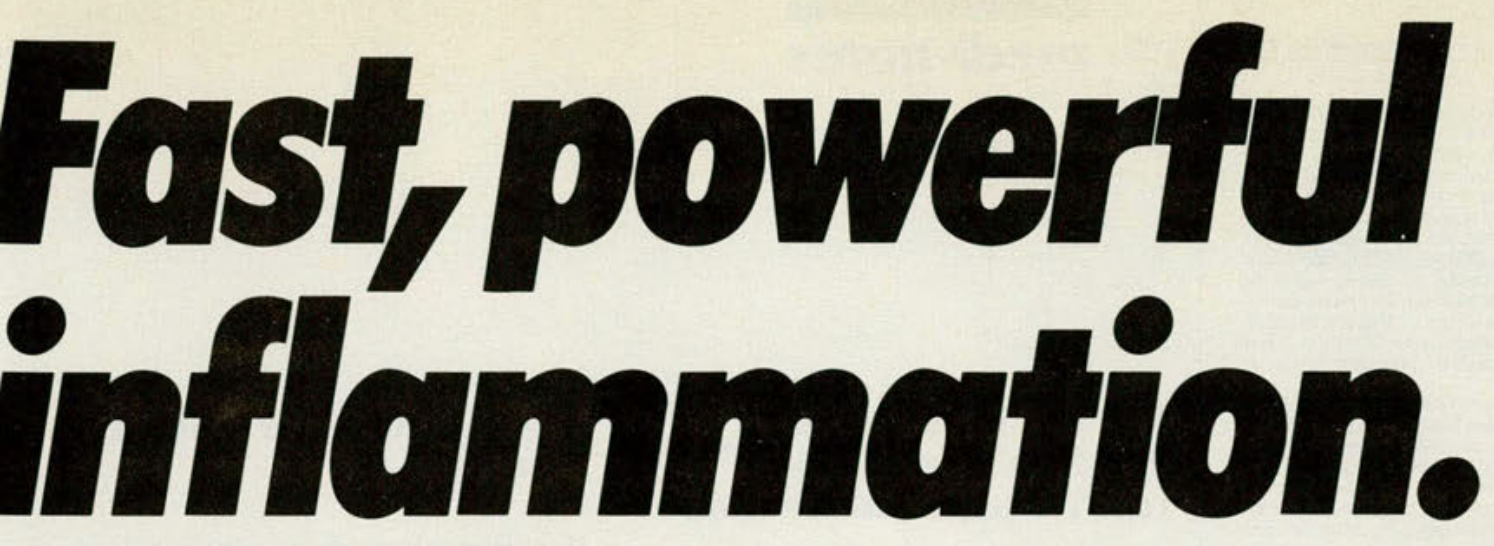

\section{Sprains}

Musculoskeletal trauma
ANAPROX Double Strength gives you fastacting, powerful, pain relief as effective as the codeine combinations and propoxyphene napsylate....with a potent anti-inflammatory bonus they can' $\downarrow$ offer.

- No oral analgesic works faster-Onset of pain relief may occur as fast as 20 minutes.

- Twice the strength-550 mg ANAPROX per tablet.

- Convenient T.I.D. dosage-The daily dosage of $1650 \mathrm{mg}$ can be used for limited periods when a higher level of analgesic/anti-inflammatory activity is required. Do not exceed this daily dose.

- Low abuse potential-No narcotic-type CNS side effects.*

\section{The Sprains \& Strains Specialist}

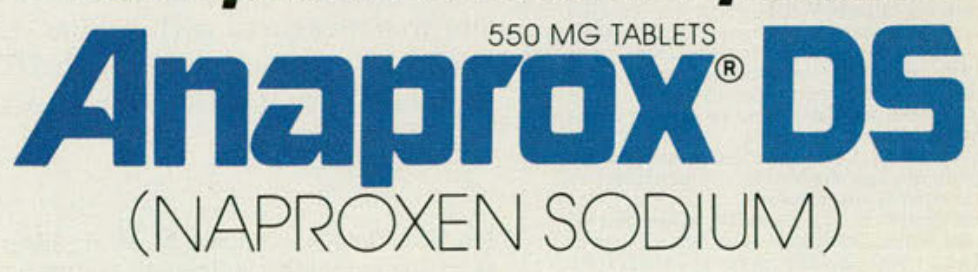

$\checkmark$ SYNTEX

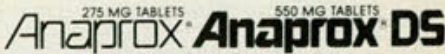

SYNTEX PUERTO RICO INC HUMACAO PR 0066

(NAPROXEN SODIUM)

-The most frequent complaints with ANAPROX DS are gastrointestina.

For brief summary of prescribing information, please see last page of advertisement.

(1989 Syntex Puerto Rico, Inc. 